
\title{
25 Research Square \\ Functional Analysis of a Viral Promoter From the Strawberry Vein Banding Virus (SVBV) Chinese Isolate
}

Lei Jiang

Anhui Agricultural University

Jing Chen

Anhui Agricultural University

You-zhi Yang

Anhui Agricultural University

Rui Li

Anhui Agricultural University

Shuang Li

Anhui Agricultural University

Zhan-qi Wang

Huzhou University

Tong Jiang ( $\sim$ jiangtong4650@sina.com )

Anhui Agricultural University

\section{Research Article}

Keywords: SVBV, Chinese isolate, full-length promoter, promoter activity, CaMV 35S promoter

Posted Date: September 7th, 2021

DOl: https://doi.org/10.21203/rs.3.rs-845742/v1

License: (9) (i) This work is licensed under a Creative Commons Attribution 4.0 International License.

Read Full License 


\section{Abstract}

\section{Background}

Promoter is an important factor during gene expression in cells. In this study, we cloned a full-length promoter from the strawberry vein banding virus (SVBV) Chinese isolate and produced several its deletion mutants.

\section{Methods}

The full-length promoter of SVBV (SP1) and its three deletion mutants (SP2, SP3, and SP4) were amplified using polymerase chain reaction (PCR). The expression activities controlled by the SVBV SP1, SP2, SP3, and SP4 were evaluated using $\beta$-glucuronidase (GUS) and green fluorescent protein (GFP) reporter genes.

Results

Our transient expression assays showed that the SVBV SP1 promoter as well as its three deletion mutants all expressed the reporter genes, but to very different levels. Interestingly, the expression activity driven by the SP1 promoter was much higher than that shown by the CaMV 35 S promoter. After stable transformation of a GUS gene into Nicotiana tabacum plants, the transgene expression level driven by the SVBV SP1 promoter was about 2.6-fold greater than that driven by the CaMV 35S promoter. In addition, the GUS gene expression levels could be enhanced by co-infiltrating the plants with the SP1 promoterdriven vector carrying the GUS gene and the vector expressing the SVBV ORF V or ORF VI.

\section{Conclusions}

The SVBV Chinese isolate promoter SP1 is a stronger promoter than the CaMV 35 S and FLt-US promoter, may be more useful for production of stable transgenic plants.

\section{Background}

Cauliflower mosaic virus (CaMV) 35S promoter is known as a strong foreign gene expression promoter and has been successfully used to study gene functions in many dicotyledonous and monocotyledonous species through Agrobacterium-mediated or particle bombardment-mediated transformation technologies. Using this plant virus derived promoter, numerous transgenic crop plants, including cotton, soybean, rice, and many other species, with improved resistance against insects, diseases, or abiotic stresses have been produced [1-6].

CaMV 35S promoter is currently the most wildly used gene expression promoter for plant stable transformation, including strawberry. Although earlier studies had shown that the CaMV 35 S promoter had strong foreign gene expression abilities in plants, recent studies have indicated that its expression activity was less effective compared with several plant derived expression promoters [7-10]. For 
example, strawberry plants transformed with an RNA silencing vector expressing an antisense fragment of a pectate lyase gene through a $35 \mathrm{~S}$ promoter showed only $30 \%$ reduction of the endogenous pectate lyase gene expression in strawberry plants, leading to a no clear phenotype change in fruit color, size, shape, and weight [7]. Owens et al. reported that they expressed a cold-inducible transcription factor, $C B F 1$, in strawberry plants [10]. Analysis of two stable transgenic strawberry lines through quantitative RT-PCR showed that the expression level of the transgene driven by the $35 \mathrm{~S}$ promotor was very weak in both leaves and receptacles. Consequently, the transgenic plants did not show a clear improvement of cold tolerance [10]. In 2001, Zhang et al. published a transformation protocol for strawberry. In that study, the authors transformed strawberry variety 'Tudla' driven by the $35 \mathrm{~S}$ promoter, only $50 \%$ of transgenic plants had $\beta$-glucuronidase (GUS) activity [9].

The level of foreign gene expression in transgenic plant is mainly depended on the strength of the promoter in the expression vector. Promoters are recognized by RNA polymerases followed by the initiation of the gene transcription. The $35 \mathrm{~S}$ promoter was originally identified in the CaMV genome, a virus known to infect mainly Cruciferae plants. Although both Strawberry vein banding virus (SVBV) and CaMV belong to the genus Caulimovirus, family Caulimoviridae [11-13], SVBV infects mainly strawberry and causes yellow vein banding and leaf twisting symptoms $[11,12,14]$. Genome of SVBV is a circular double stranded DNA of about 8 kilobases $(\mathrm{kb})$ long and contains seven open reading frames (ORFs) [12]. The arrangement of SVBV genome structure resembles that of CaMV. Sequence of SVBV promoter is also similar to the CaMV 35S promoter, and is located downstream of the ORV VI. In 2000, Wang et al. cloned the full-length SVBV promotor and used it to express a full-length infectious clone of zucchini yellow mosaic virus in cucumber, melon, squash and watermelon [15], In 2004, Pattanaik et al. further characterized the promoter from a SVBV US isolate and found that a $371 \mathrm{bp}$ fragment from the 3 ' half (352 to +19 from the transcription start site) of the promoter was able to give the maximal transcription activity during protoplast assays [16]. In the current study, we cloned the full-length promoter from the SVBV Chinese isolate (refer to SVBV SP1 thereafter), compared its expression activity with the promoter of the SVBV US isolate, and analyzed the key elements inside the promoter for their responsibility to enhance gene expression. We propose to use this promoter for foreign gene expression in transgenic strawberry or other Rosaceae plants. Our results shown here also provided an evidence showing that both SVBV ORF V and VI products can enhance foreign gene expression driven by the SVBV SP1 promoter in plants.

\section{Methods}

\section{Construction of expression vectors}

SVBV Chinese isolate DNA was isolated from an SVBV-infected strawberry leaf sample. Promoter of this virus isolate and its three deletion mutants were made through PCR amplification using viral DNA specific primers (Table 1). The PCR-amplified fragments were cloned individually into a pGEM-T vector (Promega, Madison, USA). After DNA sequencing, the correct fragments were released from the pGEM-T cloning vectors through double digestion with $B a m H I$ and $X b a l$ or $E c o R I$ and $B a m H I$ restriction enzymes. The 
double digested fragments were then inserted individually into the $B a m \mathrm{HI} / X$ bal or $E c o R I / B a m H I$ sites within the binary vector pINT121 or pCHF3 to generate pINT-SP1, pINT-SP2, pINT-SP3, pINT-SP4 or pCHFSP1, pCHF-SP2, pCHF-SP3, pCHF-SP4 construct. The pINT constructs contained a GUS gene and the pCHF constructs contained a green fluorescent protein (GFP) gene. For comparison, a full-length SVBV-E3 promoter was PCR-amplified from the plasmid pSVBV-E3 (a gift from Prof. Stenger DC, University of Nebraska, Lincoln, USA) using primer pairs FLtUSCH-F-1/FLtUSCH-R-1 or FLtUSCH-F-2/FLtUSCH-R-2 (Table 1). The resulting fragments were also inserted into the vector pINT121 or pCHF3 to produce pINTFLt-US or pCHF-FLt-US. The expression vectors were transformed individually into $A$. tumefaciens strain EHA105 cells as described previously [17] and used for the transient or stable expression assays. 
Table 1

Primer pairs used in this study.

\begin{tabular}{|c|c|c|}
\hline Name of primers & Sequence of primers & Restriction site \\
\hline $\mathrm{P} 1 / \mathrm{F}$ & 5'-GGATCCGTCAtcgcataTGTTCGAGACC-3' & BamHI \\
\hline $\mathrm{P} 1 / \mathrm{R}$ & 5'-TCTAGAATGTAAGCAGttaggccCTGTG-3' & Xbal \\
\hline $\mathrm{P} 2 / \mathrm{F}$ & 5'-GGATCCcatggactccttgactatgtaca-3' & BamHI \\
\hline $\mathrm{P} 2 / \mathrm{R}$ & 5'-TCTAGAccgGCAGttcttgactaggacct-3' & Xbal \\
\hline P3/F & 5'-GGATCCGTCAtccaaagagcacttaGACC-3' & BamHI \\
\hline P3/R & 5'-TCTAGAcgtagctacgtaccccgatggc-3' & Xbal \\
\hline P4/F & 5'-GGATCC GTCAtcgcataTGTTCGAGACC-3' & EcoRI \\
\hline $\mathrm{P} 4 / \mathrm{R}$ & 5'-TCTAGAATGTAAGCAGttaggccCTGTG-3' & BamHI \\
\hline USPCH/F-1 & 5'-GGATCCagagcacttccaaaga-3' & BamHI \\
\hline USPCH/R-1 & 5'-TCTAGAGttaggTAAGCAGCTA-3' & Xbal \\
\hline $\mathrm{CHPCH} / \mathrm{F} 1$ & 5'-GaattcGTCAtccaaagagcacttaGACC-3' & EcoRI \\
\hline $\mathrm{CHPCH} / \mathrm{R} 1$ & 5'-GGATCCcgtagctacgtaccccgatggc-3' & BamHI \\
\hline $\mathrm{CHPCH} / \mathrm{F} 2$ & 5'-Gaattccatggactccttgactatgtaca-3' & EcoRI \\
\hline $\mathrm{CHPCH} / \mathrm{R} 2$ & 5'-GGATCCccgGCAGttcttgactaggacct-3' & BamHI \\
\hline $\mathrm{CHPCH} / \mathrm{F} 3$ & 5'-GaattcGTCAtccaaagagcacttaGACC-3' & EcoRI \\
\hline $\mathrm{CHPCH} / \mathrm{R} 3$ & 5'-GGATCC cgtagctacgtaccccgatggc-3' & BamHI \\
\hline $\mathrm{CHPCH} / \mathrm{F} 4$ & 5'-GaattcGTCAtcgcataTGTTCGAGACC-3' & EcoRI \\
\hline $\mathrm{CHPCH} / \mathrm{R} 4$ & 5'-GGATCCATGTAAGCAGttaggccCTGTG-3' & BamHI \\
\hline USPCH/F-2 & 5'-Gaattcagagcacttccaaaga-3' & EcoRI \\
\hline USPCH/R-2 & 5'-GGATCCGttaggTAAGCAGCTA-3' & BamHI \\
\hline GUS/F & 5'-CATGGCTGGATATGTATCACCGCGT-3' & - \\
\hline GUS/R & 5'-CGAAGTTCATGCCAGTCCAGCGT-3' & - \\
\hline$\beta$-actin/F & 5'-CAATCCAGACACTGTACTTTCTCTC-3' & - \\
\hline$\beta$-actin/R & 5'-AAGCTGCAGGTATCCATGAGACTA-3' & - \\
\hline
\end{tabular}

\section{Transient gene expression assays and stable plant transformation}


Transient gene expression assays were conducted through Agrobacterium-infiltration as described [18]. Briefly, Agrobacterium containing one of the expression vectors was grown till $\mathrm{OD}_{600}=0.8$. The Agrobacterium cells were pelleted and then resuspended in an infiltration solution (10 mM 2morpholinoethane-sulfonic acid, $10 \mathrm{mM} \mathrm{MgCl}_{2}$ and $100 \mu \mathrm{M}$ acetosyringone) till $\mathrm{OD}_{600}=1.5$. Agrobacterium suspension $(500 \mu \mathrm{L})$ harboring a specific expression vector was infiltrated into a leaf or injected into a stem of a $N$. benthamiana plant using one $\mathrm{ml}$ syringes. The infiltrated leaves were harvested at $64 \mathrm{~h}$ post infiltration (hpi) and analyzed individually for GUS activities using a fluorometric assay or for GFP signals under a confocal microscope. For Agrobacterium-injected stems, stem cross sections were cut just above the injection site and used for the histochemical staining assays. Three independent experiments were conducted for each construct. Agrobacterium-mediated stable transformation of $N$. tabacum was performed using a leaf disc transformation method as previously reported [19]. Positive transgenic plants were selected on a MS medium containing $100 \mu \mathrm{g} / \mathrm{mL}$ kanamycin and $500 \mu \mathrm{g} / \mathrm{mL}$ carbenicillin, followed by PCR confirmation of the selected plants using GUS or GFP gene specific primers.

\section{Analyses of GUS gene expression using histochemical and fluorometric assays}

Histochemical assay was performed as described previously [20] with minor modifications. Briefly, freehand cut sections were made from plant roots, stems, or leaves, and then incubated overnight in a GUS staining solution (1 mM 5-bromo-4-chloro-3-indolyl- $\beta$-D-glucuronic acid in a $50 \mathrm{mM}$ sodium phosphate buffer, $\mathrm{pH} 7.0$ ) at $37^{\circ} \mathrm{C}$. Sections (4-6 $\mu \mathrm{m}$ thick) were cut from the embedded tissues using an 11800 LKB Pyramitome (LKB-BROMMA, Stockholm, Sweden). Images of the sections were captured using a Leica DC300 stereomicroscope (Leica, Mannheim, Germany). For fluorometric assays, N. benthamiana leaves were sampled and homogenized in the Passive Lysis Buffer (Promega, Wisconsin, USA). After centrifugation, supernatants were collected and used for the fluorometric assays as described previously [21]. Fluorescence from 4-methylumbelliferone (MU), a cleavage product from 4-methylumbelliferyl- $\beta$-Dglucuronide (MUG), was measured using a luminescence Perkin-Elmer LS50B spectrometer with excitation and emission wavelengths set at 365 and $455 \mathrm{~nm}$, respectively. Protein contents in these samples were estimated using an Eppendorf BioPhotometer (Eppendorf, Hamburg, Germany). GUS activity in each sample was calculated as the amount of MU released from MUG in p moles, per minute, per microgram of protein. The mean GUS activity in the pINT121 vector-transformed tissues was considered as $100 \%$ and used to normalize the GUS activities from other tissue samples. The statistical difference between different samples were analyzed using the LSD method in the SPSS 12.0 software.

\section{Quantitative RT-PCR (qRT-PCR) analysis}

Total RNA was isolated from transgenic tobacco seedlings harboring the pINT-SP1, pINT-FLt-US or pINT121 expression vector using the TRIzol Reagent (Invitrogen, CA, USA) followed by DNase I treatment. The first strand cDNA was synthesized using $1 \mathrm{mg}$ total RNA per $20 \mu \mathrm{L}$ RT reaction, and an AMV RNA PCR Kit (TaKaRa, Dalian, China). qPCR reaction was performed using a SYBR Premix Ex Tap ${ }^{\text {TM }}$ II kit as instructed (TaKaRa, Dalian, China). Expression level of the GUS gene in the transgenic tobacco lines was 
determined by qRT-PCR using gene specific primers (Table 1). The expression level of tobacco $\beta$-actin gene was used as an internal control during the assay as described previously [18].

\section{Co-infiltration of N. benthamiana leaves with the SVBV SP1 promoter and one of the SVBV protein expression constructs}

Individual ORFs of the SVBV Chinese isolate were PCR-amplified and cloned individually behind a CaMV 35 S promotor inside the pBIN-PLUS vector to generate pBIN-ORFI, pBIN-ORFII, pBIN-ORFIII, pBIN-ORFIV,

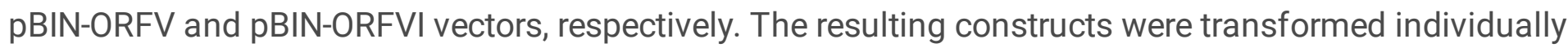
into A. tumefaciens cells and then co-infiltrated into $N$. benthamiana leaves with an Agrobacterium harboring the pINT-SP1 vector. Activities of the GUS gene in these infiltrated leaves were determined at 64 hpi though the fluorometric assay as described by Zhang et al. [21].

\section{Results}

\section{Construction of expression vectors harboring the full-length or partial SVBV promoters}

Full-length SVBV promoter was PCR-amplified from the SVBV Chinese isolate DNA (GenBank accession number HE681085). Sequence analysis using the Plant CARE program [22] showed that the full-length SVBV promoter contains several typical cis-acting elements like TATA box, CAAT box, and potential cisregulatory elements including GATA motifs and TC-rich repeats (Ni et al. 2011). According to this prediction, a full-length SVBV Chinese isolate promoter (e.g., SP1) and three of its deletion mutants (SP2, SP3, and SP4) were made (Fig. 1A). The position of SP1 promoter in SVBV genome is indicated. Mutant SP2 promoter contained a fragment of SP1, ranging from nucleotides $-324 \mathrm{bp} \sim+1$, and retained only the downstream core promoter region with a GA-motif and two CAAT-boxes. Mutant SP3 promoter had a deletion from nucleotide position $-984 \sim-819 \mathrm{bp}$ and thus represented the upstream regulatory elements plus the core promoter region. Mutant SP4 promoter contained the full-length promoter sequence except the 30 nucleotides upstream of the transcription initiation site $(+1)$. The full-length and mutant SVBV promoters were individually used to replace the $35 \mathrm{~S}$ promoter in the PCHF3 or pINT121 expression vector to produce pCHF-SP1, pCHF-SP2, pCHF-SP3, pCHF-SP4, pINT-SP1, pINT-SP2, pINT-SP3 and pINT-SP4, respectively. The expression vector pCHF3 and pINT121 without their original 35S promoter (e.g., pCHF3$35 \mathrm{~S} \Delta$ and pINT121-35S $\Delta$ ) were served as the negative control vectors. In this study, the full-length promoter of the SVBV American isolate (SP-US) was also PCR-amplified and used to replace the 35S promoter in pCHF3 and pINT121 to generate pCHF-FLt-US and pINT-FLt-US, respectively. These two vectors were also used in this study for comparisons (Fig. 1B,C).

The full-length SVBV SP1 promoter has a greater potential for transient expression of exogenous genes in plants 
Transient expression of a GFP gene in N. benthamiana stems. Agrobacterium harboring expression vector pCHF-SP1, pCHF-SP2, pCHF-SP3, pCHF-SP4, pCHF-FLt-US or pCHF3-35S $\Delta$ was injected into $N$. benthamiana stems. The injected stems were harvested at $64 \mathrm{hpi}$ and freehand cut cross sections from these stems were examined under a Leica DC300 stereomicroscope. Results of the study showed that GFP green fluorescence appeared exclusively in the vascular tissues in the stem sections injected with expression vector pCHF-SP1, pCHF-SP2, pCHF-SP3, pCHF-SP4 or pCHF-FLt-US. No green fluorescence was observed in the sections from the plants injected with Agrobacterium harboring pCHF3-35S $\triangle$ (Fig. 2A).

Histochemical detection of transiently expressed GUS gene in stem sections. Agrobacterium harboring expression vector pINT-SP1, pINT-SP2, pINT-SP3, pINT-SP4, pINT-FLt-US or pINT-35S $\Delta$ was injected into stems of $N$. benthamiana plants. Histochemical staining of the sections from the paraffin embedded stem tissues followed by Light Microscopy showed that blue staining signal, representing GUS gene expression, was mainly localized in the vascular tissues as well as in some cells in the cortex of all pINTSP1-, pINT-SP2-, pINT-SP3-, pINT-SP4- or pINT-FLt-US-injected stem sections. No blue staining was observed in the sections from the stems injected with Agrobacterium harboring the pINT-35S $\Delta$ vector (Fig. 2B).

Analysis of GUS activity in tissues through fluorometric assays. Agrobacterium harboring vector pINTSP1, pINT-SP2, pINT-SP3, pINT-SP4, pINT-FLt-US or pINT-35S $\triangle$ was infiltrated into $N$. benthamiana leaves. The infiltrated leaves were harvested at $3 \mathrm{dpi}$ and analyzed for GUS activities using a fluorometric assay. Results showed that the average GUS activity in the leaves infiltrated with Agrobacterium harboring the pINT-SP1 vector was about 3.2- and 1.8-fold greater than that in the leaves infiltrated with Agrobacterium harboring the pINT121 or pINT-FLt-US vector, respectively (Fig. 2C). The mean GUS activity in the leaves infiltrated with Agrobacterium harboring the mutant pINT-SP4 vector was about 1.4-fold greater than that in the leaves infiltrated with Agrobacterium harboring the pINT121 vector, but was only about $75 \%$ of that in the leaves infiltrated with Agrobacterium harboring the pINT-FLt-US vector. The mean GUS activities in the leaves infiltrated with Agrobacterium harboring the mutant pINT-SP2 or pINT-SP3 vector were lower than that in the leaves infiltrated with Agrobacterium harboring the pINT121 vector.

\section{The full-length SVBV SP1 promoter has a greater potential for stable expression of exogenous genes in plants}

Analysis of GUS activity in tissues of transgenic tobacco. Strength of promoter activity decides the expression levels of transgenes in plant. To compare the expression strength between the SVBV SP1 promoter and the SVBV US strain promoter (FLt-US) or with the CaMV 35S (35S) promoter, tobacco plants were stably transformed with pINT-SP1, pINT-FLt-US, pINT121 or pINT-35S $\Delta$ vector. Transgenic tobacco seedlings or leaves were then harvested and analyzed for the promoter expression activities though histochemical staining. Result of the experiment showed that both SVBV SP1 and FLt-US promoters gave stronger GUS gene expression in the transgenic tobacco seedlings and the fully expended leaves than that in the pINT121-transformed tobacco (Fig. 3A,B). Histochemical staining also showed that the GUS 
gene expression was in mainly in the vascular bundles. Histochemical staining also showed that all the transgenic tobacco plants showed GUS gene expression in the elongation zone of the roots (Fig. 3C). Histochemical staining of stem cross sections from the transgenic plants showed that the GUS gene expression was mainly in the epidermal layer, pith, cortex, and vascular cells (Fig. 3D). Results of this experiment suggested that the expression strength of SVBV SP1 promoter was greater than that of SVBV FLt-US promoter or CaMV 35 S promoter.

\section{Analysis of GUS activity and accumulation of GUS mRNA in transgenic expression plants}

To confirm the expression activity of SVBV SP1 promoter is stronger than that of the SVBV FLt-US promoter or CaMV 35S promoter, leaves were harvested from plants transformed with the pINT-SP1, pINTFLt-US or pINT121 vector and analyzed for GUS activity through a fluorometric assay. Result of the study showed that the average GUS activity in the leaves harvested from the plants transformed with the pINTSP1 vector was about 1.7- and 3.1-fold greater than that in the leaves harvested from the plants transformed with the pINT-FLt-US or pINT121 vector (Fig. 4A). This finding agreed with the GUS staining results shown in Fig. 3, and suggested that the promoter of the SVBV China isolate might be used to generate stable transgenic plants with significantly higher levels of transgene expressions than that in the plants transformed with a vector harboring the SVBV FLt-US or CaMV 35S promoter.

To confirm the GUS activity observed in Fig. 4A, we further determined the GUS mRNA levels in different transgenic plants using qRT-PCR. Total RNA was isolated from tobacco seedlings transformed with the pINT-SP1, pINT-FLt-US, pINT121 or pINT-35S $\triangle$ vector, and analyzed by qRT-PCR using GUS gene specific primers. Result of the assay showed that the GUS mRNA accumulated to a significantly higher level in the pINT-SP1-transformed tobacco seedlings than that in the pFLt-US- or pINT121-transformed seedlings (Fig. 4B). This result agreed with the result from the GUS activity assay shown in Fig. 4A, and indicated that the transcription activity of SVBV SP1 promoter is indeed stronger than that shown by the SVBV FLtUS promoter or the CaMV 35 S promoter.

\section{Influence of other SVBV-encoded proteins on SP1 promoter activity}

SVBV gene specific primers (Table 1) were used to amplify full-length ORF I, II, III, IV, V and VI from the SVBV Chinese isolate DNA, respectively. The lengths of the PCR products were $986 \mathrm{bp}$ (ORF I), $485 \mathrm{bp}$ (II), 320 bp (III), 1415 bp (IV), 2099 bp (V), and 1556 bp (VI). The resulting PCR fragments were individually cloned into an expression vector, pBIN438 with a 35 S promoter, to generate $\mathrm{pBIN-ORFI,} \mathrm{pBIN-ORFII,} \mathrm{pBIN-}$ ORFIII, pBIN-ORFIV, pBIN-ORFV and pBIN-ORFVI, respectively. These expression vectors were transformed individually into Agrobacterium and then co-infiltrated, individually, with the Agrobacterium harboring pINT-SP1 into $N$. benthamiana leaves. Leaves co-infiltrated with Agrobacterium harboring pINT-SP1 and pBIN438 were used as controls. Analysis of GUS activities in these Agrobacterium-infiltrated leaves showed that the GUS activities in the leaves co-infiltrated with Agrobacterium harboring PINT-SP1 and pBIN-ORFV or Agrobacterium harboring pINT-SP1 and pBIN-ORFVI were 2.7- and 2.4-fold greater than that in the leaves co-infiltrated with Agrobacterium harboring pINT-SP1 and pBIN438 (Fig. 5). GUS activities in 
the leaves co-infiltrated with Agrobacterium harboring pINT-SP1 and pBIN-ORFI, pINT-SP1 and pBIN-ORFII, pINT-SP1 and pBIN-ORFIII or pINT-SP1 and pBIN-ORFIV were similar to those in the leaves co-infiltrated with Agrobacterium harboring pINT-SP1 and pBIN438. These results indicated that SVBV ORF V and VI could enhance foreign gene expression driven by the SVBV SP1 promoter while other SVBV ORFs had no significant effect on foreign gene expression driven by this promoter.

\section{Discussion}

\section{Transient gene expression driven by SVBV SP1 promoter}

In this study we compared the strength of gene expression driven by the full-length SP1 promoter with its several deletion mutants. Our results indicated that the SP2 promoter containing $324 \mathrm{bp}$ and one GAmotif and two CAAT-boxes was able drive the expression of a GFP or a GUS gene in plant. This finding suggests that this $324 \mathrm{bp}$ fragment is likely the minimal region for the promoter activity. The SP3 promoter retained the minimal promoter region and several upstream regulatory elements (e.g., five CAATboxes and two TAAT-boxes). The expression levels driven by the SP3 promoter was about 3-fold greater than that of SP2 promoter. This result indicated that the five CAAT-boxes and two TAAT-boxes in the SP3 promoter had significantly influence on the promoter activity. The SP1 promoter has two additional CAATboxes compared with the SP3 promoter at its 5 ' end and its expression strength was about 3-fold greater than the SP3 promoter, indicating that the additional two CAAT-boxes at the 5 ' end of SP1 promoter also had strong effect on the SVBV promoter. The TATA-box sequence at $40 \mathrm{bp}$ upstream of the transcription initiation site (+1) is known as a binding site for RNA polymerase II [23]. In this study, we deleted the 3' end 30 nucleotides from the SP1 promoter and found that this deletion mutant (i.e., SP4) had much lower promoter activity than that of the SP1 promoter. This finding suggested that deletion of the 3 ' end 30 nucleotides from the SP1 promoter might affect the binding of RNA polymerase II and lead to a weak promoter activity.

\section{Stable gene expression in plant driven by the SVBV promoter}

The CAAT box and TAAT-box are what is known as a core promoter, also known as the basal promoter or simply the promoter [24-27]. The SP1 promoter cloned in this study possess seven CAAT-boxes and four TAAT-boxes, and its expression activity is likely controlled by these regulatory elements. It was reported that the constitutive expression driven by promoters may be controlled by some special regulatory elements. For example, the regulatory element as-2-box in the CaMV 35S promoter could be induced by light to promote the expression of genes in leaf photosynthetic tissues and to induce the expression of genes in roots $[28,29]$. The GATA-box in the cassava vein mosaic virus promoter was shown to regulate the expression of genes in green tissues [30,31]. The as-2-box and GATA-box sequences are also present in the SVBV SP1 promoter. We speculate that the constitutive gene expression driven by the SP1 promoter may also be regulated by these two regulatory elements. In this study, our qRT-PCR results showed that 
the expression levels of the GUS reporter gene driven by the SP1 promoter was significantly higher than that driven by the CaMV $35 S$ promoter.

\section{Effects of other SVBV-encoded proteins on SVBV SP1 promoter activity}

Both SVBV and CaMV are members in the genus Caulimovirus. Based on the classification of CaMV ORFs, the ORF I of SVBV encodes a protein necessary for virus intercellular movement. The ORF II encodes a protein important for aphid vector transmission, and the ORF III encodes a non-sequence specific DNA binding protein. The ORF IV encodes a capsid protein (CP) that is important for virion formation. The ORF V encodes a reverse transcriptase and the ORF VI encodes a viral determinant of disease symptoms and host range. The function of the ORF VII encoded protein remained unclear [3234]. Our Agrobacterium-infiltration results showed that co-infiltration of the ORF V or ORF VI vector with the SP1 promoter driven expression vector resulted in an enhanced GUS gene expression, but the other ORF expression vectors had no such effect.

CaMV first transcribes RNA with the virion DNA as the template, and then reverse transcribe double stranded DNA using above RNA as the template [35]. ORF V is the replicase gene, its encoding protein plays a role in DNA synthesis from RNA during virus reverse transcription. ORF V can promote the transcription activity of the promoter, it is possible that the replicase protein encoded by ORF $\mathrm{V}$ accelerates the replication of the plasmid containing the full-length promoter of Chinese isolate. increasing the required amount of template for transcribing mRNA, resulting in increased expression of reporter gene GUS.

CaMV gene ORF VI is a multifunctional protein, which has trans activation function. As a trans-activator (TAV), it forms dense cell inclusions in the virus infected cytoplasm [36,37]. The translation of ORF VII followed by ORF VI is reinitiated by CaMV 35S RNA through the ribosome diverting mechanism [38], with the assistance of TAV, increasing translation by 2-3 times. SVBV ORF VI encodes a TAV [39] and its presence may promote transcriptional activity of the promoter and enhance the expression of reporter gene GUS.

\section{Conclusions}

CaMV 35S promoter is currently the most widely used expression promoter during plant stable transformation. But the SVBV Chinese isolate promoter SP1 is a stronger promoter than the CaMV 35S and FLt-US promoter, may be more useful for production of stable transgenic plants.

\section{Abbreviations}

CaMV: Cauliflower mosaic virus; CP: capsid protein; FLt-US: SVBV US strain promoter; GFP: green florescence protein; GUS: $\beta$-glucuronidase; kb: kilobases; MUG: methylumbelliferyl- $\beta$-D-glucuronide; ORFs: 
open reading frames; qRT-PCR: quantitative RT-PCR; SVBV: strawberry vein banding virus; TAV: transactivator.

\section{Declarations}

\section{Acknowledgments}

Not applicable.

\section{Authors' contributions}

TJ and LJ conceived and designed the study and wrote the paper. LJ, JC, YY, RL, SL, and ZW performed the experiments and analyzed the data. All authors read and approved the final manuscript.

\section{Funding}

This work was supported by grants from the National Natural Science Foundation of China (No. 32072386 and 31801700), the Anhui Provincial Key Research and Development Plan (202004a06020013) and the Postdoctoral Science Foundation of Anhui Province (No. 2019B360).

\section{Availability of data and materials}

All data generated or analyzed during this study are included in this published article.

\section{Ethics approval and consent to participate}

Not applicable.

\section{Consent for publication}

Not applicable.

\section{Competing interests}

The authors declare that they have no competing interests.

\section{Author details}

${ }^{1}$ School of Plant Protection, Anhui Agricultural University, Hefei, 230036, People's Republic of China. ${ }^{2}$ Key Laboratory of Vector Biology and Pathogen Control of Zhejiang Province, College of Life Sciences, Huzhou University, Huzhou 313000, People's Republic of China. ${ }^{3}$ Anhui Province Key Laboratory of Integrated Pest Management on Crops, Hefei, 230036, People's Republic of China. ${ }^{4}$ Key Laboratory of Biology and Sustainable Management of Plant Diseases and Pests of Anhui Higher Education Institutes, Anhui Agricultural University, Hefei, 230036, People's Republic of China. 


\section{References}

1. Holtorf S, Apel K, Bohlman H. Comparision of different constitutive and inducible promoters for the overexpression of transgenes in Arabidopsis thaliana. Plant Mol Biol. 1995;29(4):637-646.

2. Mitsuhara I, Ugaki M, Hirochika H, Ohshima M, Murakami T, et al. Efficient promoter cassettes for enhanced expression of foreign genes in dicotyledonous and monocotyledonous plants. Plant Cell Physiol. 1996;37(1):49-59.

3. Nagadhara D, Ramesh S, Pasalu IC, Rao YK, Krishnaiah NV, et al. Transgenic indica rice resistant to sap-sucking insects. Plant Biotechnol J. 2003;1(3):231-240.

4. Lim S, Nam M, Kim KH, Lee SH, Moon JK, et al. Development of a new vector using Soybean yellow common mosaic virus for gene function study or heterologous protein expression in soybeans. J Virol Methods. 2016;228:1-9.

5. Kiselev KV, Aleynova OA, Ogneva ZV, Suprun AR, Dubrovina AS. 35S promoter-driven transgenes are variably expressed in different organs of Arabidopsis thaliana and in response to abiotic stress. Mol Biol Rep. 2021;48(3):2235-2241.

6. Li H, Yang Y, Wang H, Liu S, Jia F, et al. The receptor-like kinase ERECTA confers improved water use efficiency and drought tolerance to poplar via modulating stomatal density. Int J Mol Sci. 2021;22(14):7245.

7. James DJ, Passey AJ, Easterbrook MA, Solomon MG, Barbara DJ. Progress in the introduction of transgenes for pest resistance in apples and strawberry. Phytoparasitica. 1992;20(Suppl):83-87.

8. Graham J, Mcnicol RJ, Greig K. Towards genetic based insect resistance in strawberry using the cowpea trypsin inhibitor gene. Ann Appl Biol. 1995;127(1):163-173.

9. Zhang ZH, Wu LP, Dai HY, Wang GY, Bi XY. Regeneration and transformation in vitro of the strawberry varieties. Acta Horticulturae Sinica. 2001;28(3):189-193.

10. Owens CL, lezzoni AF, Hancock JF. Enhancement of freezing tolerance of strawberry by heterologous expression of CBF1. Acta Hort. 2003;626:93-100.

11. Petrzik K, Benes V, Mráz I, Honetslegrová-Fránová J, Ansorge W, et al. Strawberry vein banding virusdefinitive member of the genus Caulimovirus. Virus Genes. 1998;16(3):303-305.

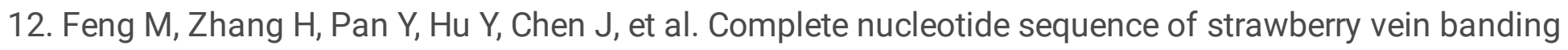
virus Chinese isolate and infectivity of its full-length DNA clone. Virol J. 2016;13(1):164.

13. Dickison V, MacKenzie TDB, Singh M, Lawrence J, Nie X. Strawberry vein banding virus isolates in eastern Canada are molecularly divergent from other isolates. Arch Virol. 2017;162(6):1777-1781. 
14. Stenger DC, Mullin RH, Morris TJ. Isolation, molecular cloning and detection of strawberry vein banding virus DNA. Phytopathology. 1988;78(2):154-159.

15. Wang Y, Gaba V, Wolf D, Xia XD, Zelcer A, et al. Identification of a novel plant virus promoter using a potyvirus infectious clone. Virus Genes. 2000;20(1):11-17.

16. Pattanaik S, Dey N, Bhattacharyya S, Maiti IB. Isolation of full-length transcript promoter from the Strawberry vein banding virus (SVBV) and expression analysis by protoplasts transient assays and in transgenic plants. Plant Sci. 2004;167(3):427-438.

17. Lin Q, Wu F, Sheng P, Zhang Z, Zhang X, et al. The SnRK2-APC/C(TE) regulatory module mediates the antagonistic action of gibberellic acid and abscisic acid pathways. Nat Commun. 2015;6:7981.

18. Zhong X, Wang ZQ, Xiao R, Cao L, Wang Y, et al. Mimic phosphorylation of a $\beta C 1$ protein encoded by TYLCCNB impairs its functions as a viral suppressor of RNA silencing and a symptom determinant. $J$ Virol. 2017;91(16):e00300-17.

19. Naqvi RZ, Asif M, Saeed M, Asad S, Khatoon A, et al. Development of a triple gene Cry1Ac-Cry2AbEPSPS construct and its expression in Nicotiana benthamiana for insect resistance and herbicide tolerance in plants. Front Plant Sci. 2017;8:55.

20. Jefferson RA, Kavanagh TA, Bevan MW. GUS fusions: $\beta$-glucuronidase as a sensitive and versatile gene fusion marker in higher plants. EMBO J. 1987;6(13):3901-3907.

21. Zhang H, Hou J, Jiang P, Qi S, Xu C, et al. Identification of a 467 bp promoter of maize phosphatidylinositol synthase gene (ZmPIS) which confers high-level gene expression and salinity or osmotic stress inducibility in transgenic tobacco. Front Plant Sci. 2016;7:42.

22. Lescot M, Déhais $P$, Thijs $G$, Marchal $K$, Moreau $Y$, et al. PlantCARE: a database of plant cis-acting regulatory elements and a portal to tools for in silico analysis of promoter sequences. Nucleic Acids Res. 2002;30(1):325-327.

23. Yang Y, Li R, Qi M. In vivo analysis of plant promoters and transcription factors by agroinfiltration of tobacco leaves. Plant J. 2000;22(6):543-551.

24. Mantovani R. The molecular biology of the CCAAT-binding factor NF-Y. Gene. 1999;239(1):15-27.

25. Smale ST, Kadonaga JT. The RNA polymerase II core promoter. Annu Rev Biochem. 2003;72:449-479.

26. Brand A, Quimbaya M, Tohme J, Chavarriaga-Aguirre P. Arabidopsis LEC1 and LEC2 orthologous genes are key regulators of somatic embryogenesis in cassava. Front Plant Sci. 2019;10:673.

27. Liu N, Xu S, Yao Q, Zhu Q, Kai Y, et al. Transcription factor competition at the y-globin promoters controls hemoglobin switching. Nat Genet. 2021;53(4):511-520. 
28. Schindler U, Cashmore AR. Photoregulated gene expression may involve ubiquitous DNA binding proteins. EMBO J. 1990;9(11):3415-3427.

29. Foster E, Hattori J, Labbé $H$, Ouellet T, Fobert PR, et al. A tobacco cryptic constitutive promoter, tCUP, revealed by T-DNA tagging. Plant Mol Biol. 1999;41(1):45-55.

30. Verdaguer B, de Kochko A, Fux Cl, Beachy RN, Fauquet C. Functional organization of the Cassava vein mosaic (CsVMV) promoter. Plant Mol Biol. 1998;37(6):1055-1067.

31. Andrieu A, Breitler JC, Siré C, Meynard D, Gantet P, et al. An in planta, Agrobacterium-mediated transient gene expression method for inducing gene silencing in rice (Oryza sativa L.) leaves. Rice (NY). 2012;5(1):23.

32. Mahmoudpour A. Infectivity of recombinant strawberry vein banding virus DNA. J Gen Virol. 2003;84(Pt 6):1377-1381.

33. Haas G, Azevedo J, Moissiard G, Geldreich A, Himber C, et al. Nuclear import of CaMV P6 is required for infection and suppression of the RNA silencing factor DRB4. EMBO J. 2008;27(15):2102-2112.

34. Feng $M$, Zuo D, Jiang $X$, Li S, Chen J, et al. Identification of strawberry vein banding virus encoded P6 as an RNA silencing suppressor. Virology. 2018;520:103-110.

35. Hull R, Covey SN, Maule AJ. Structure and replication of Caulimovirus genomes. J Cell Sci Suppl. 1987;7:213-229.

36. Rothnie HM, Chapdelaine Y, Hohn T. Pararetroviruses and retroviruses: a comparative review of viral structure and gene expression strategies. Adv Virus Res. 1994;44:1-67.

37. Yasaka R, Nguyen HD, Ho SY, Duchêne S, Korkmaz S, et al. The temporal evolution and global spread of Cauliflower mosaic virus, a plant pararetrovirus. PLoS One. 2014;9(1):e85641.

38. Ryabova LA, Hohn T. Ribosome shunting in the cauliflower mosaic virus 35S RNA leader is a special case of reinitiation of translation functioning in plant and animal systems. Genes Dev. 2000;14(7):817829.

39. Li S, Hu Y, Jiang L, Rui P, Zhao Q, et al. Strawberry vein banding virus P6 protein is a translation transactivator and its activity can be suppressed by FvelF3g. Viruses. 2018;10(12):71.

\section{Figures}


A

$\begin{array}{llll}-984 & -819 & -324 & -30+1\end{array}$

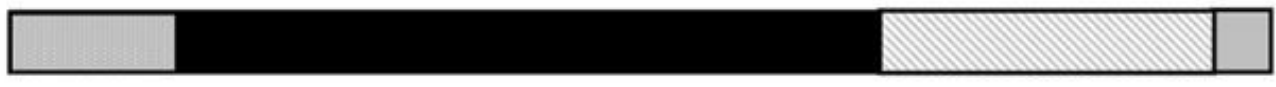

SP1

SP2

SP3

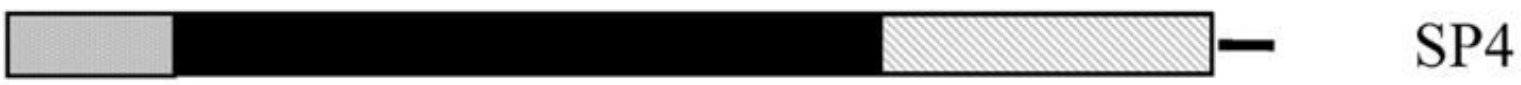

B

$-964+1$

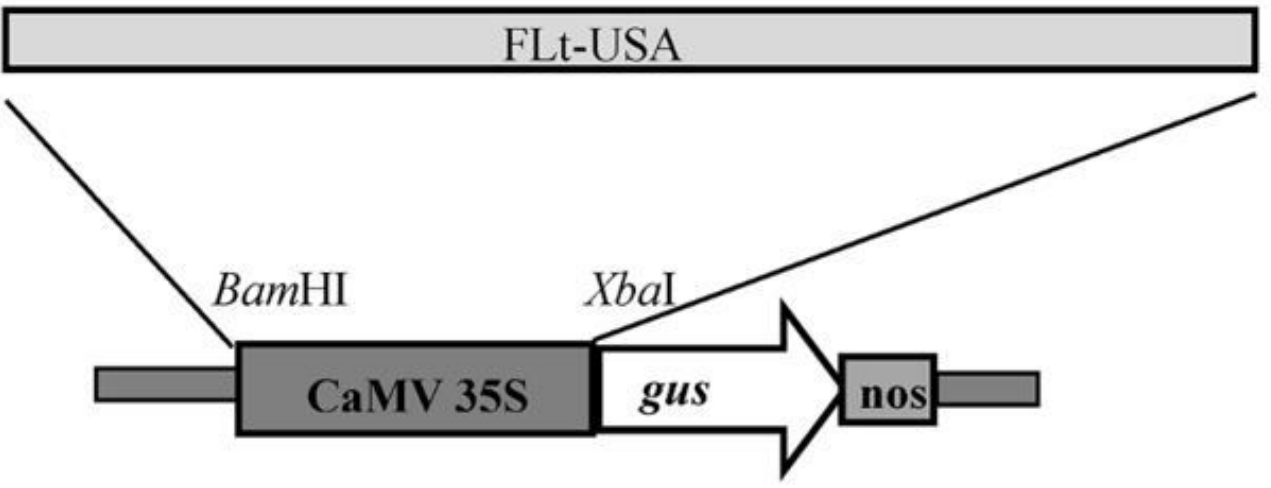

SP-US

pINT121

C

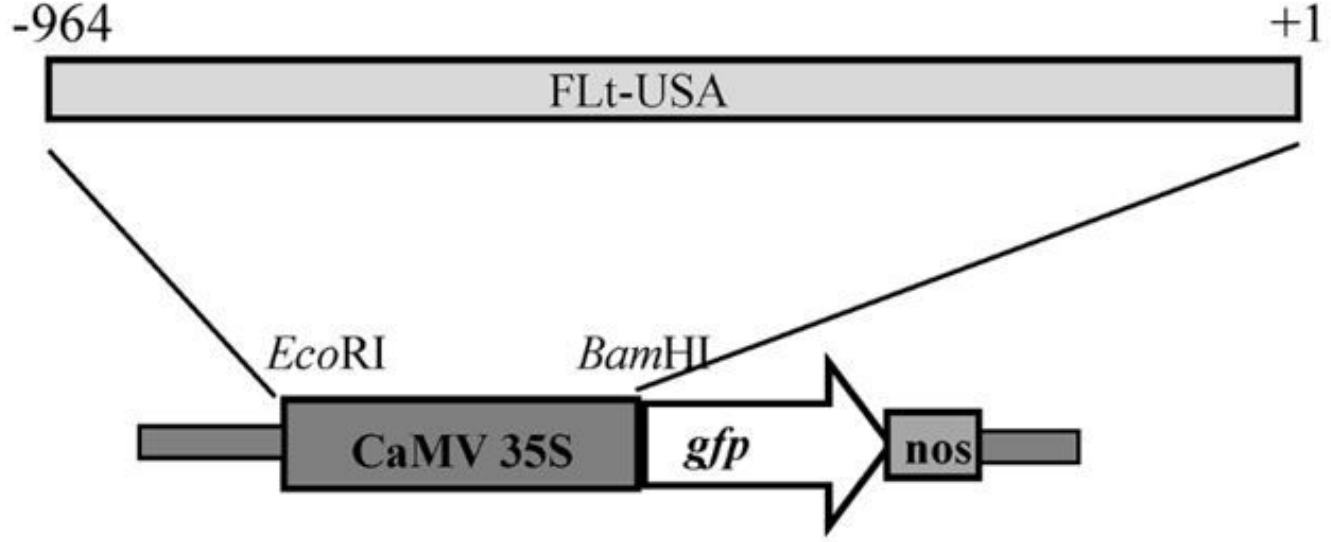

SP-US

pCHF3

Figure 1

Schematic representations of the expression vectors. (A) The full-length SVBV SP1 promoter and its deletion mutants. (B) Schematic illustration of the pINT-FLt-US. (C) Schematic illustration of the pCHFFLt-US. 


\section{A}
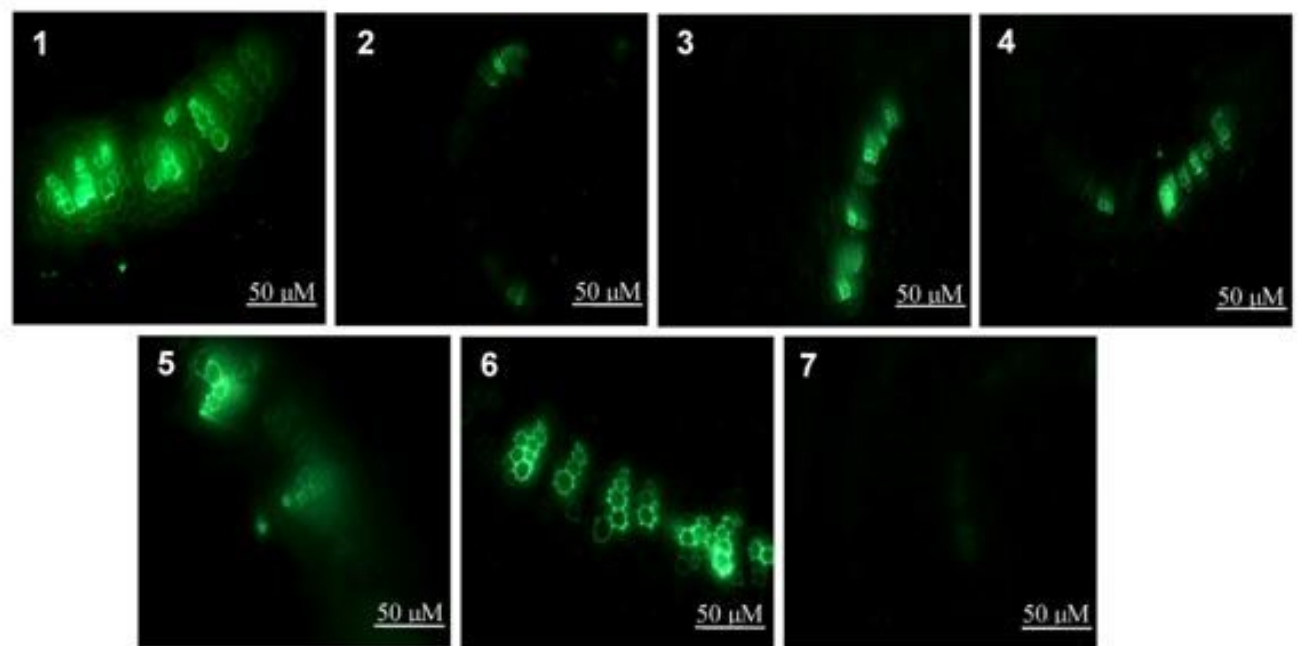

B

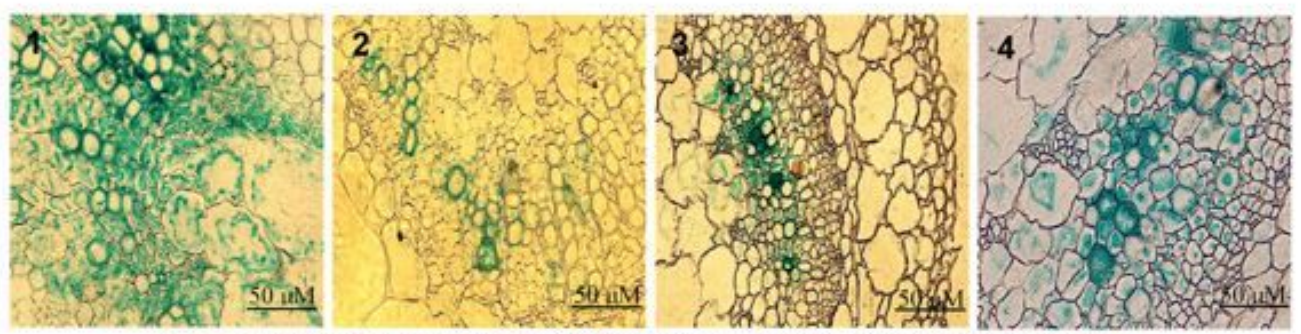

5

6

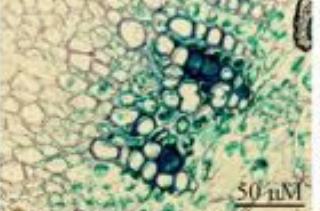

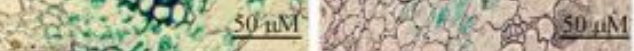

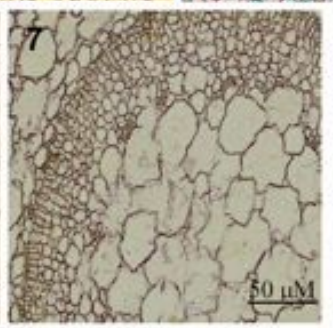

C

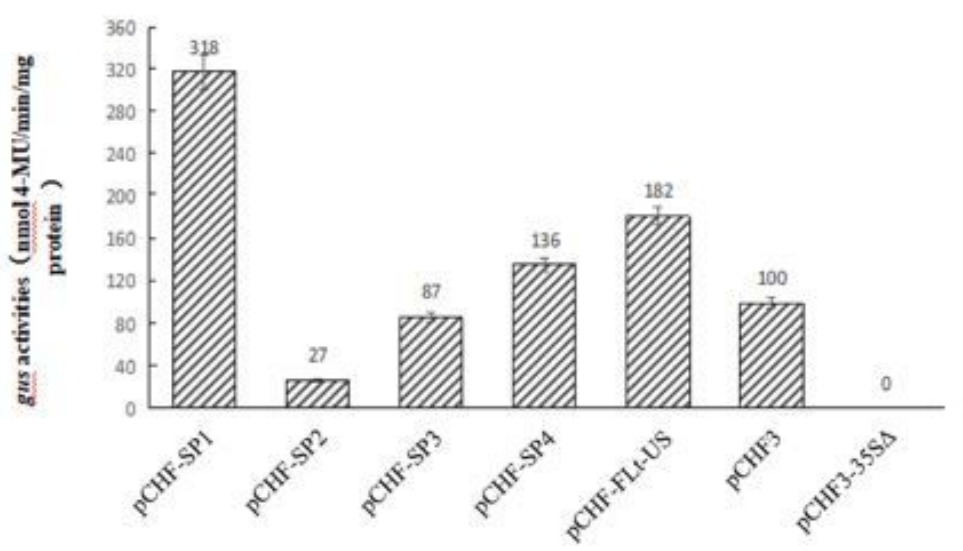

\section{Figure 2}

The full-length SVBV SP1 promoter has a greater potential for expressing exogenous genes in transient expression plants. (A) Transient expression of GFP in N. benthamiana stems. Agrobacterium harboring the pCHF-SP1 (1), pCHF-SP2 (2), pCHF-SP3 (3), pCHF-SP4 (4), pCHF-FLt-US (5), pCHF3 (6), or pCHF3$35 \mathrm{~S} \Delta(7)$ vector was injected into the stems of N. benthamiana plants. Freehand sections were cut from the injected stems at $64 \mathrm{hpi}$ and examined for GFP signal under a fluorescent microscope. Bar $=50 \mu \mathrm{M}$. 
(B) Analysis of GUS gene expression in N. benthamiana stems. Agrobacterium harboring the pINT-SP1 (1), pINT-SP2 (2), pINT-SP3 (3), pINT-SP4 (4), pINT-FLt-US (5), pINT121 (6), or pINT-35S $\Delta$ (7) vector was injected into the stems of $\mathrm{N}$. benthamiana plants. The injected stems were harvested at $64 \mathrm{hpi}$ and stained overnight in a $1 \mathrm{mM}$ X-Gluc staining solution prior to paraffin embedding. Sections prepared from the embedded tissues were examined under a microscope for GUS staining results. Blue staining indicates positive expressions of the GUS gene in stems. Bar $=50 \mu \mathrm{M}$. (C) Analysis of GUS activity in N. benthamiana leaves. Agrobacterium harboring the pINT-SP1, pINT-SP2, pINT-SP3, pINT-SP4, pINT-FLt-US, pINT121, or pINT-35S $\triangle$ (negative control) vector was infiltrated into leaves of $\mathrm{N}$. benthamiana plants. The infiltrated leaves were harvested at $64 \mathrm{hpi}$ and used for GUS activity fluorometric assays. Each treatment had five biological replicates and the experiment was repeated three times. Standard errors were determined using the LSD method in the SPSS 12.0 software. 
A
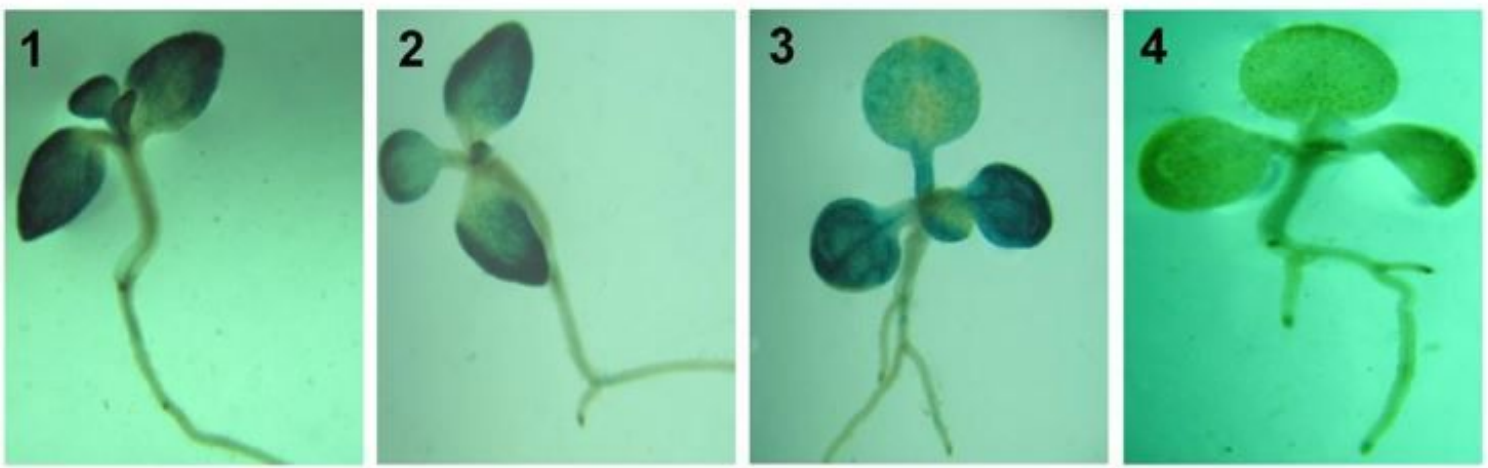

B
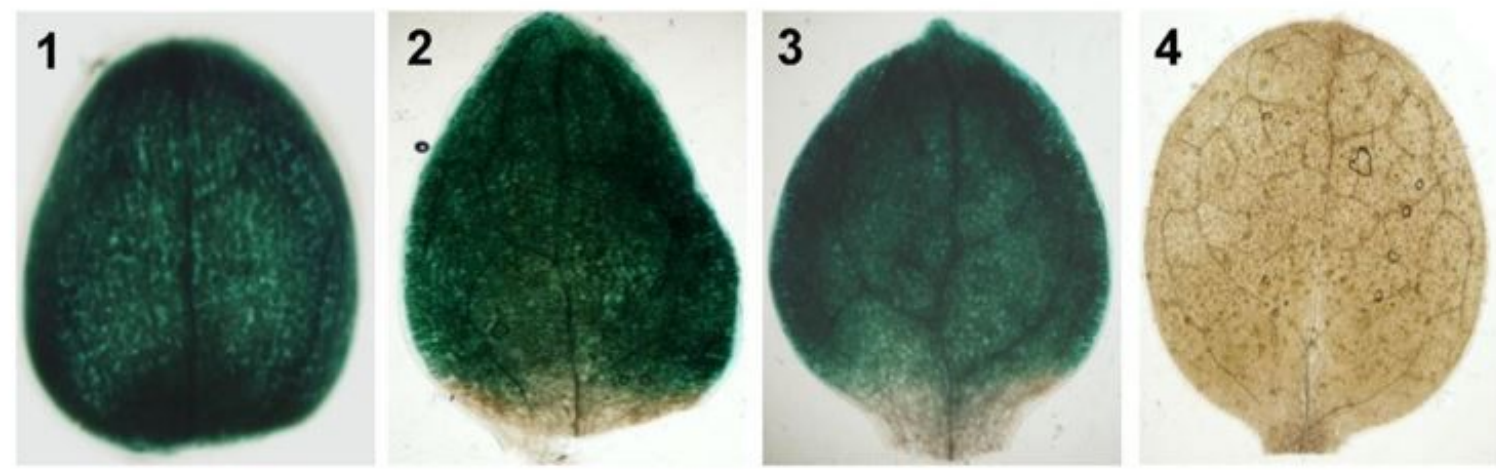

C
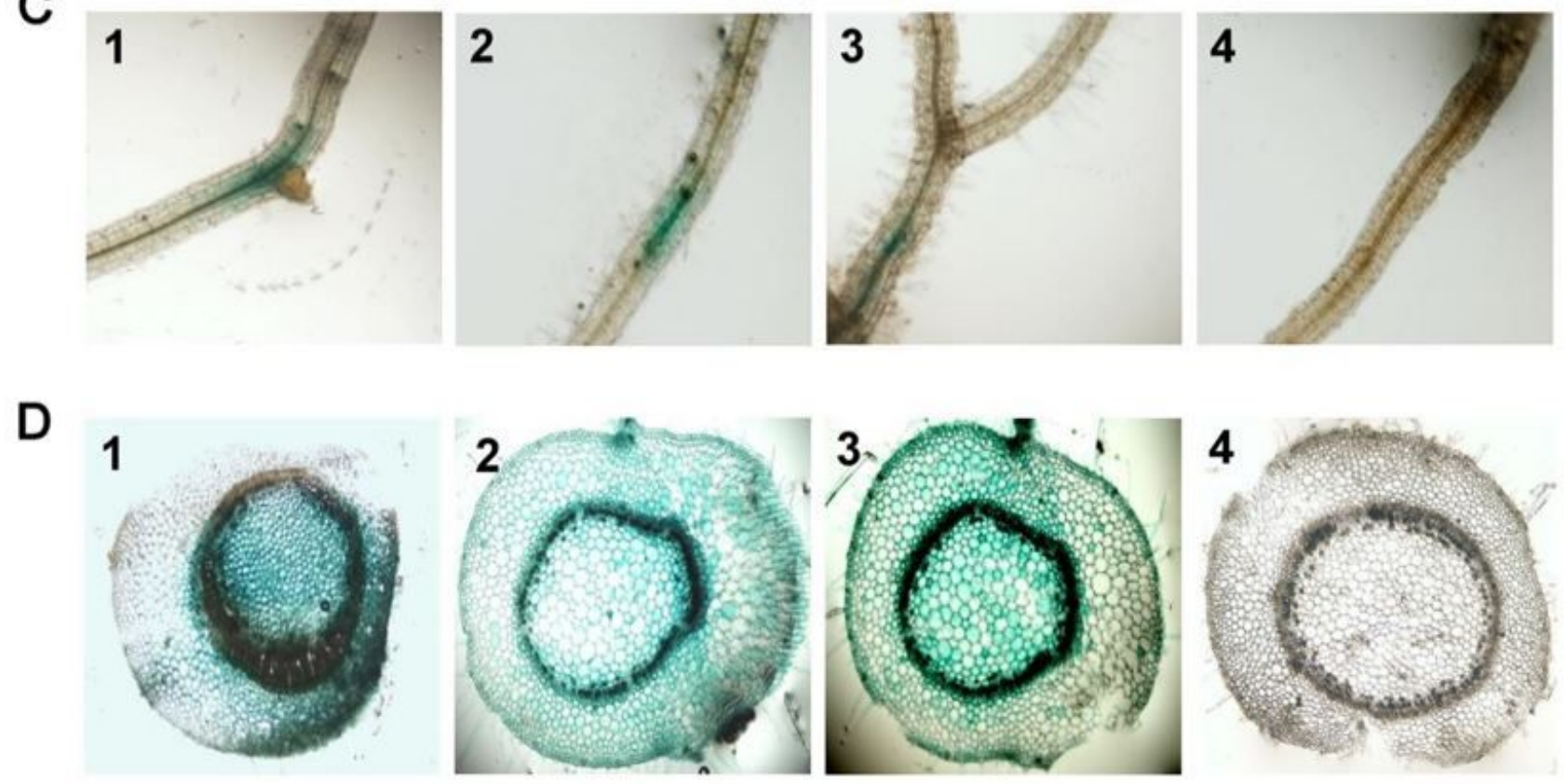

\section{Figure 3}

Histochemical assays of GUS gene expression in transgenic tobacco seedlings, leaves, roots, and stems. N. benthamiana plants transformed with the pINT SP1 (1), pINT-FLt-US (2), pINT121 (3) or pINT-35S $\Delta$ (4, negative control) vector were used in these assays. Expression of the GUS gene in these transgenic plant tissues were determined by overnight tissue staining in a $1 \mathrm{mM}$ X-Gluc staining solution. (A) Images of seedlings representing the four different transgenic plant lines are shown. (B) Images of fully expended 
leaves were harvested from different transgenic plants and stained for GUS activities. (C) Images of roots were harvested from different transgenic plants and stained for GUS activities. (D) images of stem cross sections were prepared from different transgenic plants and stained for GUS activities.

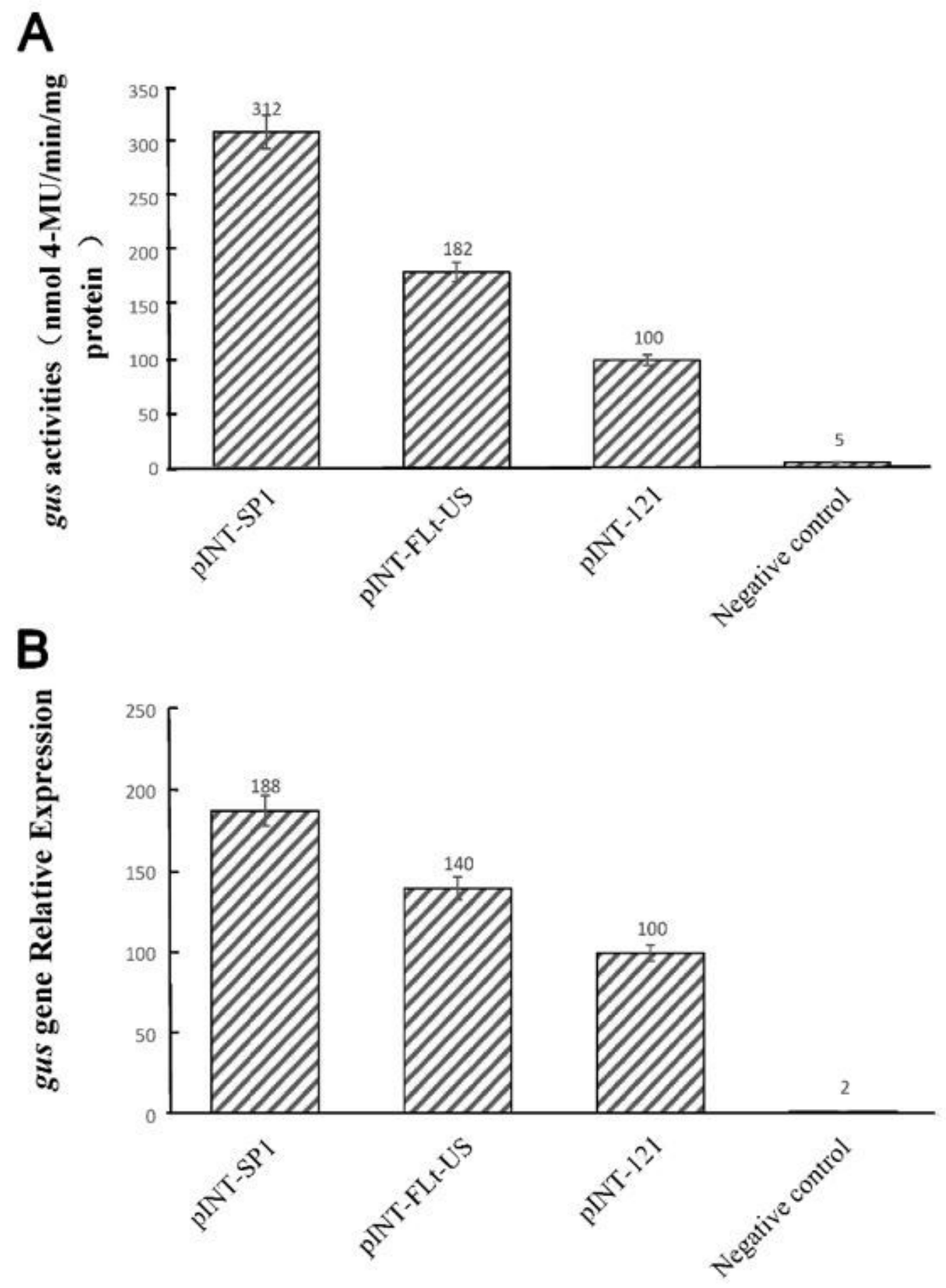

Figure 4

Analysis of GUS activity and accumulation of GUS mRNA in transgenic expression plants. (A) GUS activities in the leaves from different transgenic tobacco plants. Leaves were harvested from tobacco 
plants transformed with pINT-SP1, pINT-FLt-US, pINT121 or pINT-35S $\Delta$ (negative control) vector. The harvested leaf tissues were analyzed for GUS activities through a fluorometric assay. Each treatment had five biological replicates and the experiment was repeated three times. Standard errors were determined using the LSD method in the SPSS 12.0 software. (B) Relative expression levels of GUS gene in different transgenic tobacco plants. Total RNA was extracted from leaves harvested from tobacco plants transformed with the pINT-SP1, pINT-FLt-US, pINT121 or pINT-35S $\triangle$ (negative control) vector. Relative GUS mRNA level in each sample was determined by qRT-PCR using GUS gene specific primers. Each treatment had five biological samples and the experiment was repeated three times. Relative expression level of tobacco $\beta$-actin gene was used as an internal control. Standard errors were determined using the LSD method in the SPSS 12.0 software.
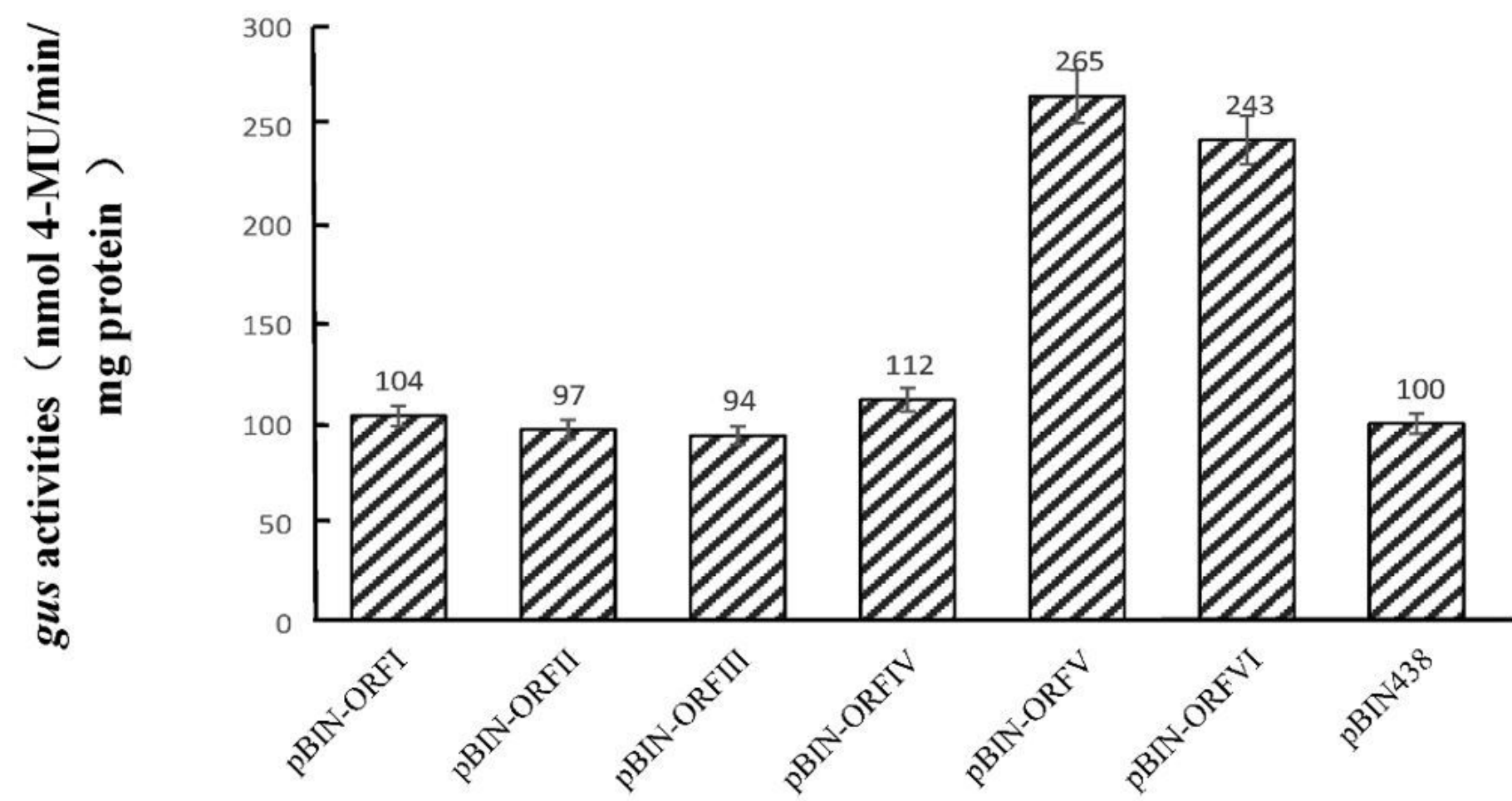

\section{Figure 5}

Effects of SVBV-encoded proteins on foreign gene expression driven by the SVBV SP1 promoter. Agrobacterium harboring the pINT-SP1 vector was co-infiltrated with Agrobacterium harboring the pBINORFI, pBIN-ORFII, pBIN-ORFIII, pBIN-ORFIV, pBIN-ORFV, pBIN-ORFVI, or pBIN438 (control) vector into N. benthamiana leaves. The infiltrated leaves were harvested at $64 \mathrm{hpi}$ and then analyzed for GUS activities through a fluorometric assay. Each treatment had five biological samples and the experiment was repeated three times. Standard errors were determined using the LSD method in the SPSS 12.0 software. 